\title{
Mitochondrial metabolism and the control of vascular smooth muscle cell proliferation
}

\section{Mario Chiong ${ }^{1 *}$, Benjamín Cartes-Saavedra ${ }^{1}$, Ignacio Norambuena-Soto ${ }^{1}$, David Mondaca-Ruff ${ }^{1}$, Pablo E. Morales ${ }^{1}$, Marina García-Miguel ${ }^{1}$ and Rosemarie Mellado ${ }^{2}$}

${ }^{1}$ Faculty of Chemical and Pharmaceutical Sciences, Advanced Center for Chronic Diseases, University of Chile, Santiago, Chile

2 Faculty of Chemistry, Pontifical Catholic University of Chile, Santiago, Chile

\section{Edited by:}

Vasu D. Appanna, Laurentian

University, Canada

Reviewed by:

Mitsuo Tagaya, Tokyo University of Pharmacy and Life Sciences, Japan Xin Qi, Case Western Reserve University, USA

*Correspondence:

Mario Chiong, Advanced Center for Chronic Diseases, Facultad Ciencias Químicas y Farmacéuticas,

Universidad de Chile, Sergio

Livingstone $P 1007$

Santiago 8380492, Chile

e-mail:mchiong@uchile.cl
Differentiation and dedifferentiation of vascular smooth muscle cells (VSMCs) are essential processes of vascular development. VSMC have biosynthetic, proliferative, and contractile roles in the vessel wall. Alterations in the differentiated state of the VSMC play a critical role in the pathogenesis of a variety of cardiovascular diseases, including atherosclerosis, hypertension, and vascular stenosis. This review provides an overview of the current state of knowledge of molecular mechanisms involved in the control of VSMC proliferation, with particular focus on mitochondrial metabolism. Mitochondrial activity can be controlled by regulating mitochondrial dynamics, i.e., mitochondrial fusion and fission, and by regulating mitochondrial calcium handling through the interaction with the endoplasmic reticulum (ER). Alterations in both VSMC proliferation and mitochondrial function can be triggered by dysregulation of mitofusin-2, a small GTPase associated with mitochondrial fusion and mitochondrial-ER interaction. Several lines of evidence highlight the relevance of mitochondrial metabolism in the control of VSMC proliferation, indicating a new area to be explored in the treatment of vascular diseases.

\footnotetext{
Keywords: vascular smooth muscle cell, proliferation, mitofusin-2, mitochondrial metabolism, mitochondrial dynamics
}

\section{INTRODUCTION}

Vascular smooth muscle cells (VSMCs) are the main component of the artery's medial layer. These cells undergo contraction and thereby regulate blood vessel tone and consequently blood flow and pressure. VSMC contraction depends on the interaction between smooth muscle $\alpha$-actin, $\beta$-myosin heavy chain, h-caldesmon, and calponin (Rzucidlo et al., 2007; Cecchettini et al., 2011). VSMCs also possess important secretory properties that ensure synthesis and repair of extracellular matrix components and regulate the structure of the vascular wall (Cecchettini et al., 2011). Normal VSMCs are not terminally differentiated cells with very low rates of proliferation and secretion (Rzucidlo et al., 2007; Cecchettini et al., 2011). Changes in the VSMC phenotype have been extensively described in the development and progression of atherosclerosis, hypertension, and neointimal formation (Campbell and Campbell, 1985; Rzucidlo et al., 2007; Cecchettini et al., 2011). This phenotypic switching includes altered expression of contractile proteins, increased matrix production, expression of inflammatory cytokines, and production of proteases (Campbell and Campbell, 1985). The capacity for contraction, proliferation, migration, and secretion in VSMCs are affected by a wide range of factors, including mechanical forces, contractile agonists such as angiotensin II, extracellular matrix, reactive oxygen species (ROS), endothelial-VSMC interactions, platelet derived growth factor (PDGF), transforming growth factor- $\beta 1$, hypoxia, and many other growth factors (Campbell and Campbell, 1985; Cecchettini et al., 2011). As a result, VSMCs constitute basic structural and functional elements in the artery wall and their malfunction leads to vascular disease. Recently, VSMC mitochondrial metabolism has been raised as part of novel mechanisms involved in the complex regulation of the VSMC phenotype, especially involving VSMC proliferation. In this review, we will examine the evidence that supports this hypothesis.

\section{REGULATION OF MITOCHONDRIAL METABOLISM}

Mitochondria have been considered the energy powerhouses in all eukaryotic cells (Scheffler, 2001). They are particularly abundant in muscle cells and most of the energy needed for muscle contraction is provided by mitochondrial metabolism (Kuznetsov et al., 2009). Mitochondria has four compartments: the outer mitochondrial membrane (OMM), the intermembrane space (IMS), the inner mitochondrial membrane (IMM), and the mitochondrial matrix (Scheffler, 2001). The IMM is particularly dense and enriched in a variety of membrane proteins, including the mitochondrial respiratory complexes and the ATP synthase (Scheffler, 2001). The driving energy for ATP synthesis comes from the generation of a proton motive force by the electron transport chain in the IMM. This gradient of concentration and charge constitutes the intermembrane mitochondrial potential $(\Delta \psi \mathrm{m})$ and is maintained by the oxidation of reduced substrates generated mainly by the oxidation of metabolites in the Krebs cycle and $\beta$-oxidation of fatty acids (Scheffler, 2001; Kuzmicic et al., 2011).

VSMCs exhibit unusually high rates of glucose metabolization and lactate production under normal, well-oxygenated conditions (Butler and Siegman, 1985). Under resting conditions, the 
rate of oxygen consumption and lactate production are often almost equal on a molar basis, resulting in approximately $30 \%$ of the ATP supply coming from mitochondria, but at least $90 \%$ of the flux through glycolysis resulting in lactate production (Paul, 1983). In spite of the low contribution of mitochondria to the VSMC bioenergetics, mitochondrial dysfunction, and particularly mitochondrial DNA damage, has recently been associated to atherosclerosis (Yu and Bennett, 2014). Recent studies show that during VSMC phenotypic switching, mitochondria decreases glucose oxidation and increases fatty acid oxidation (Salabei and Hill, 2013). This suggests that VSMC redirects the use of glucose or its metabolites to pathways that support the biosynthesis of DNA and other molecules required for VSMC proliferation. This process is similar to the Warburg effect present in cancer cells (Vander Heiden et al., 2009).

Mitochondria were originally thought as discrete, isolated entities scattered in the cytoplasm. However, this vision has changed to a dynamic network model in which mitochondria are in close communication with the endoplasmic reticulum (ER) and the entire morphology of the organelle is controlled by coordinated fusion and fission events. The appropriate balance of these processes is essential to maintain mitochondrial stability and function (Kuzmicic et al., 2011; Parra et al., 2011).

\section{MITOCHONDRIAL DYNAMICS AND METABOLISM}

Mitochondrial fusion stimulates the assembly of individual mitochondria that combine their membranes (Figure 1A). This process is controlled by mitofusins (Mfn) 1 and 2 and OPA-1, a member of the dynamin family of mechanoenzymes (Parra et al., 2011). Mfn-1 and Mfn-2 are two evolutionarily conserved GTPase proteins that attach to the OMM via a bipartite, $\mathrm{N}$-terminal transmembrane domain with their C-terminal coiledcoil region inserting into the cytosol. During mitochondrial fusion, Mfn-1 and Mfn-2 form hetero-oligomers that hydrolyze GTP and promote rearrangement of mitochondrial membranes (Carlucci et al., 2008). Mitochondrial fusion is a two-step process, where the IMM and OMM fuse by separate events (Malka et al., 2005; Zorzano et al., 2010), which can be explained by the mitochondrial sub-localization of Mfn-1, Mfn-2, and OPA-1. Mfn-1 and Mfn-2 are located in the OMM, where they can regulate the OMM fusion, and OPA-1 is located in the IMM, where it can regulate the IMM fusion (Zorzano et al., 2010). Mitochondrial fission entails fragmentation of tubular interconnected mitochondria into several smaller individual organelles (Figure 1B). The OMM protein FIS-1 and the GTPase dynamin related protein-1 (DRP-1) are the main elements of the mitochondrial fission machinery (Parra et al., 2011). Down regulation or inhibition of
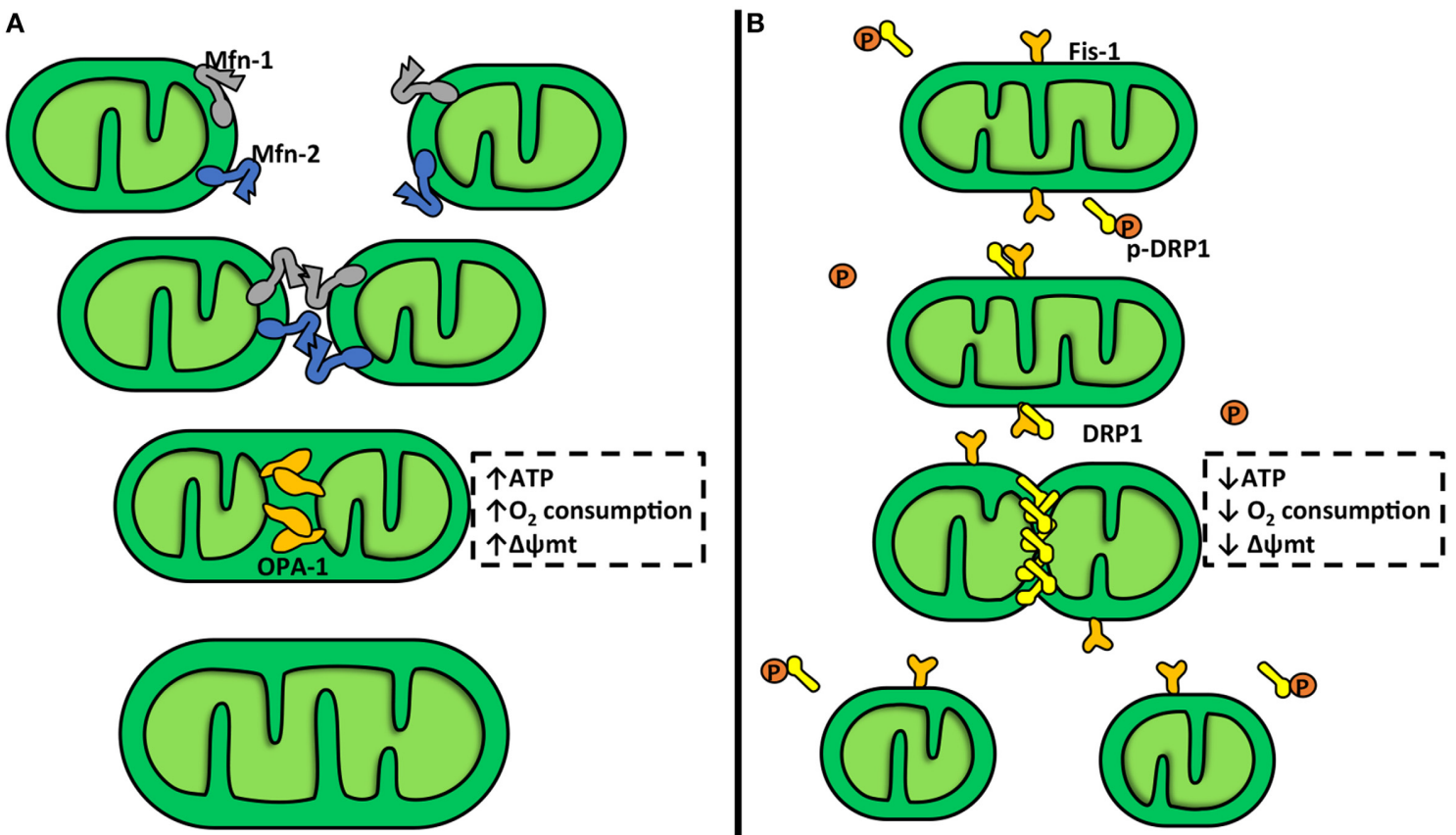

FIGURE 1 | Mitochondrial dynamics. (A) Mitochondrial fusion. This is a two-step process which involves three different proteins: mitofusin-1 and 2 (Mfn-1 and Mfn-2) and optic atrophy protein-1 (OPA-1). Mfn-1 and Mfn-2 are transmembrane GTPases embedded in the outer mitochondrial membrane (OMM). The C-terminal coiled-coil region of Mfn-1 and Mfn-2 mediates tethering between mitochondria through homo- or heterotypic complexes formed between adjacent mitochondria. This interaction mediates OMM fusion. OPA-1 is a dynamin-related protein localized in the inner mitochondrial membrane (IMM), facing the intermembrane space. OPA-1 participates in the attachment and fusion of IMM. Mitochondrial fusion is associated with an increase in the mitochondrial potential $(\Delta \psi \mathrm{m})$, oxygen consumption, and ATP production. (B) Mitochondrial fission. In this process participates dynamin-related protein-1 (DRP-1) and fission protein-1 (FIS-1). DRP-1 is a large GTPase found soluble in the cytosol of cells from where it shuttles onto and off mitochondria. DRP-1 assembles into spirals at division sites around the OMM to drive the fission process. In yeast, the mechanism for recruitment of DRP-1 to the mitochondria requires FIS-1, a tetratricopeptide domain protein anchored into and evenly coating the entire OMM. DRP-1 activity is inhibited by a protein kinase A (PKA) phosphorylation. Mitochondrial fission is associated with a $\Delta \psi \mathrm{m}$, oxygen consumption, and ATP production decrease. 
DRP-1 or FIS-1 severely inhibits mitochondrial fission and generates an extended mitochondrial membranous network (James et al., 2003; Cerveny et al., 2007). Furthermore, DRP-1 knockout mice show that DRP-1 not only affects mitochondrial fission but also affects cell proliferation generating non-viable embryos (Wakabayashi et al., 2009). Ishihara et al. also showed that neural specific DRP-1 knock-out mice dies shortly after birth as a result of brain hypoplasia (Ishihara et al., 2009). This process is accompanied with an imbalance between mitochondrial fusion and fission, and deregulation of normal cytochrome c release and caspase activation during apoptosis (Ishihara et al., 2009). DRP-1 possesses an N-terminal GTPase domain and a C-terminal GTPase effector domain, which is involved in intramolecular and intermolecular interactions and modulates the GTPase activity. Cytosolic DRP-1 is recruited to the mitochondrial surface into the fission foci by adaptor proteins, including FIS-1 (Hoppins, 2014; Mishra and Chan, 2014; Nasrallah and Horvath, 2014). FIS-1 is an integral protein of the OMM and contains a cytosolic hydrophobic tetratricopeptide repeat (TPR). The TPR is thought to interact with DRP-1 (Carlucci et al., 2008; Hoppins, 2014). At fission sites, DRP-1 forms spiral chains around membrane constriction sites. GTP hydrolysis provides energy to generate the mechanical force required for fission (Carlucci et al., 2008). There are other adaptors that can recruit DRP-1 to the mitochondria membrane, i.e., the mitochondrial fission factor (MFF) and mitochondrial dynamic proteins 49 and $51 \mathrm{kDa}$ (MiD49 and MiD51). These proteins can trigger mitochondrial fission through a FIS-1-independent process (Palmer et al., 2013).

Mitochondrial fission occurs preferentially at ER contact sites. Inverted formin 2 (INF2), a protein involved in actin polymerization and depolymerization located at the ER, has a direct effect on mitochondrial fission. INF2 facilitates both the interaction of DRP-1 with mitochondria and the fission process in an actin-dependent manner. Actin filaments appeared to accumulate between mitochondria and INF2-enriched ER membranes at constriction sites. Thus, INF2-induced actin filaments may drive initial mitochondrial constriction, which allows DRP-1-driven secondary constriction (Korobova et al., 2013).

Several lines of evidence have shown that the machinery that governs mitochondrial dynamics also participates in the temporal regulation of metabolism and cell death (Kuzmicic et al., 2011; Parra et al., 2011). The dynamic shifts in morphology coincide with a number of physiological events, such as transitions between different respiratory states and cristae remodeling during apoptosis (Parra et al., 2011). Aberrations in cristae morphology are accompanied by changes in metabolism (Parra et al., 2011; Nasrallah and Horvath, 2014), and together with the down regulation of OPA-1 or Mfn, trigger alterations of the IMM structures which leads to fragmented mitochondria with greatly reduced oxygen consumption and electrochemical potential (Chen and Chan, 2005). Mitochondria also contribute to glycolysis regulation; in cases of increased ATP demand, mitochondrial pyruvate dehydrogenase activity is up regulated, increasing glycolytic contribution to cell metabolism (Sharma et al., 2005). The first evidence of a relationship between mitochondrial morphology and function derived from studies of fusion inhibition, which leads to a reduced oxygen consumption and loss of $\Delta \psi \mathrm{mt}$ and mitochondrial DNA (Chen et al., 2003; Chen and Chan, 2005). Silencing of Mfn-2 substantially impairs metabolic parameters (Pich et al., 2005), and animal models of obesity show a marked reduction of Mfn-2 levels in muscle cells. Similarly, decreased OPA-1 levels lead to mitochondrial fragmentation, decreased oxygen consumption, and $\Delta \psi \mathrm{mt}$ dissipation, and have been implicated in the pathogenesis of neurodegenerative diseases and heart failure (Bossy-Wetzel et al., 2003; Chen et al., 2009). Taken together, these results reinforce the concept that mitochondrial plasticity is critical for cell metabolism adaptation (Soubannier and Mcbride, 2009).

\section{MITOCHONDRIA-ER COMMUNICATION AND METABOLISM}

Like mitochondrion, ER is a highly dynamic organelle, undergoing constant shape remodeling, according to the cell requirements (Bravo-Sagua et al., 2013, 2014; English and Voeltz, 2013). Furthermore, both organelles also have great intracellular motility, being able to change their distribution in the subcellular space by moving along the cytoskeleton (Boldogh and Pon, 2007; Bola and Allan, 2009). This motility is important for cell physiology, because it determines the apposition between organelles and preferential sites of signaling. Among other functions, ER is the main intracellular $\mathrm{Ca}^{2+}$ reservoir. This ion is released to the cytoplasm by two channels, the ryanodine receptor channel (RyR) and the inositol trisphosphate receptor channel (InsP3R), and is returned to the ER by the activity of the sarco/endoplasmic reticulum $\mathrm{Ca}^{2+}$ ATPase (SERCA), a pump with high affinity and velocity (Figure 2A) (Berridge et al., 2003).

$\mathrm{Ca}^{2+}$ has several roles within the mitochondria, being required for protein synthesis (Joyal et al., 1995), activation of several citric acid cycle dehydrogenases, oxidative phosphorylation, ATP synthesis (Griffiths and Rutter, 2009), and it also enhances ROS production (Dykens, 1994). The OMM, initially considered a permeable barrier, plays a critical role in mitochondrial $\mathrm{Ca}^{2+}$ uptake (Csordas et al., 2002; Rapizzi et al., 2002). $\mathrm{Ca}^{2+}$ crosses this membrane through the low affinity channel VDAC, which is regulated by chaperones (Schwarzer et al., 2002), NADH (Lee et al., 1996) and Bcl-2 family proteins (Vander Heiden et al., 2001). $\mathrm{Ca}^{2+}$ then crosses the IMM through the mitochondrial $\mathrm{Ca}^{2+}$ uniporter (mCU), driven by the $\Delta \psi \mathrm{mt}$ (Kirichok et al., 2004; Baughman et al., 2011; De Stefani et al., 2011; Rizzuto et al., 2012). $\mathrm{Ca}^{2+}$ uptake into mitochondria requires high cytoplasmatic $\mathrm{Ca}^{2+}$ levels in the surrounding area, concentrations achieved in the proximity of $\mathrm{Ca}^{2+}$ release sites located in the ER such as the InsP3R (Figure 2B) (Csordas et al., 1999; Bravo-Sagua et al., 2013; Naon and Scorrano, 2014).

We and others have shown that functional coupling between these organelles promotes efficient $\mathrm{Ca}^{2+}$ entry into mitochondria, resulting in a higher $\Delta \psi \mathrm{m}$ and oxygen consumption (Figure $2 \mathrm{~B}$ ) (Cardenas et al., 2010; Bravo et al., 2011). Increased mitochondrial $\mathrm{Ca}^{2+}$ concentration enhances oxidative phosphorylation, through the activation of four mitochondrial dehydrogenases: FAD-glycerol 3-phosphate dehydrogenase, pyruvate dehydrogenase, NAD-isocitrate dehydrogenase and oxoglutarate dehydrogenase (Denton, 2009). In fact, blocking the entry of $\mathrm{Ca}^{2+}$ into the mitochondria reduces oxidative phosphorylation and energetically compromises the cell (Cardenas et al., 2010). Therefore, 


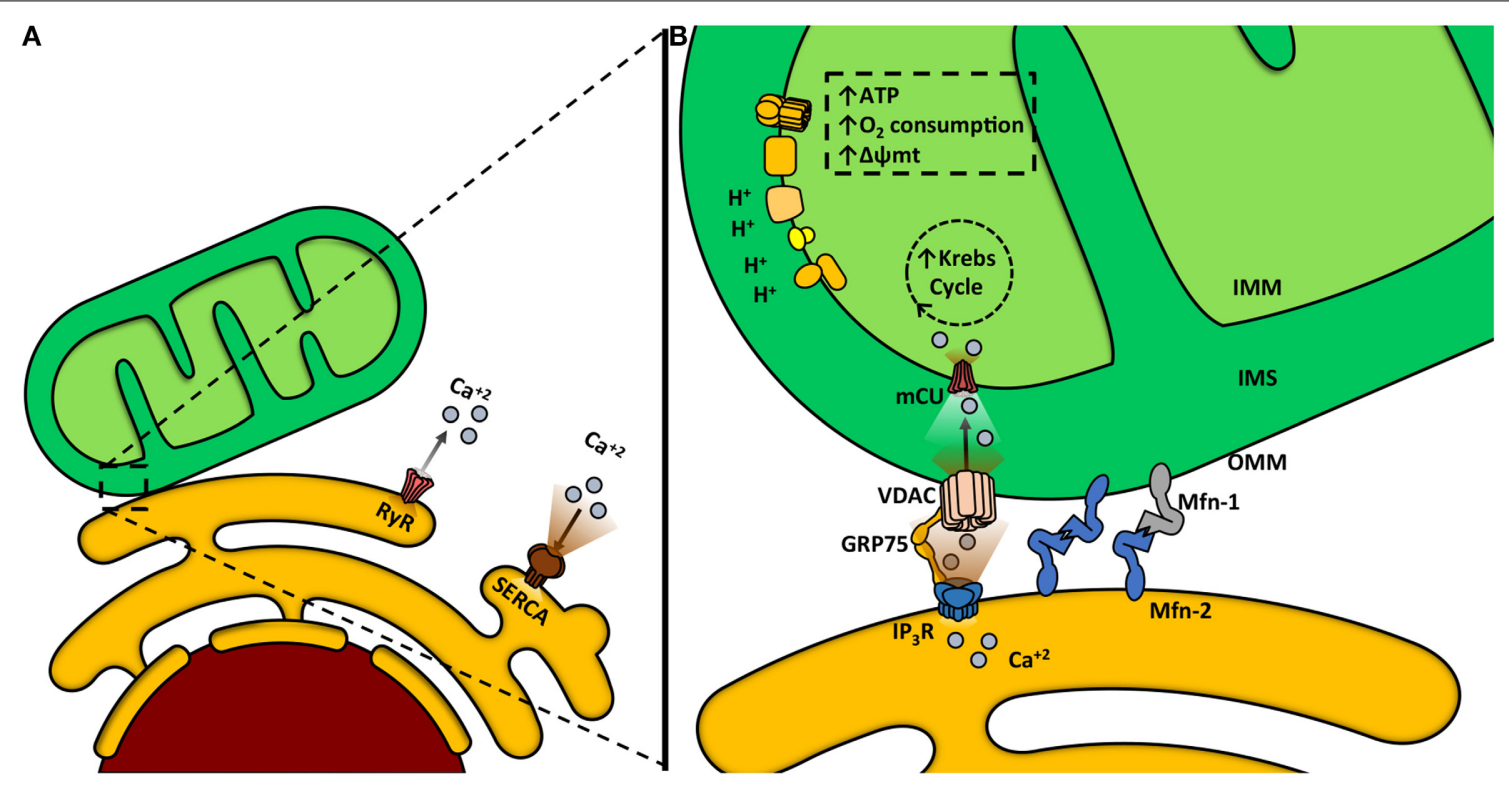

FIGURE 2 | Mitochondria-endoplasmic reticulum (ER) coupling. (A) $\mathrm{Ca}^{2+}$ release from the ER to the cytosol is mediated by two channels: ryanodine receptor channel (RyR) and inositol trisphosphate receptor channel (InsP3R). The sarcoendoplasmic reticulum $\mathrm{Ca}^{2+}$ transport ATPase (SERCA) is a pump that transports $\mathrm{Ca}^{2+}$ from the cytoplasm into the ER. (B) $\mathrm{Ca}^{2+}$ transfer from the ER to the mitochondria occurs within the mitochondria-associated membranes (MAMs). $\mathrm{Ca}^{2+}$ is released from the ER to the mitochondria through the InsP3R. Glucose-regulated protein 75 (GRP75) is a mitochondrial chaperone that mediates the molecular interaction between InsP3R with the voltage-dependent anion channel (VDAC). $\mathrm{Ca}^{2+}$ import across the OMM occurs through VDAC. $\mathrm{Ca}^{2+}$ crosses the IMM through the mitochondrial $\mathrm{Ca}^{2+}$ uniporter channel (mCU) thanks to the considerable driving force represented by the negative transmembrane potential and the high $\mathrm{Ca}^{2+}$ concentration within the intermembrane space. Increased mitochondrial $\mathrm{Ca}^{2+}$ concentration activates mitochondrial dehydrogenases and enhances the oxidative phosphorylation, increasing oxygen consumption, $\Delta \psi \mathrm{m}$ and ATP production. several dehydrogenases of the tricarboxylic acid cycle increase their activity in response to increments in mitochondrial $\mathrm{Ca}^{2+}$ content, which in turn depends on $\mathrm{Ca}^{2+}$ transients evoked by muscle contraction. This allows an appropriate coupling between contractile function and energy supply (Denton and Mccormack, 1990; Liu and O'Rourke, 2009).

The close contact zone between ER and mitochondria is called Mitochondria-Associated Membrane (MAM) (Vance, 2014). Several proteins compose and modulate this physical interaction, such as calcium channels (InsP3R, VDAC), mitochondriashaping proteins (Mfn-2), chaperones (GRP75, sigma-1 receptor, calnexin, oxidoreductases), sorting proteins (PACS-2), and other enzymes [see reviews (Rowland and Voeltz, 2012; Bravo-Sagua et al., 2013, 2014)]. The physical junction between the ER and mitochondria relies on Mfn-2 and other proteins (De Brito and Scorrano, 2008). In fact, genetic ablation of Mfn-2 results in disrupted ER-mitochondria communication and causes a decrease in both $\mathrm{Ca}^{2+}$ transfer and mitochondrial bioenergetics (Figure 3 ) (Bravo et al., 2011; Bravo-Sagua et al., 2013).

Our group described changes in mitochondrial dynamics and mitochondrial metabolism associated to cardiomyocyte apoptosis (Parra et al., 2008), protection during heart ischemia/reperfusion (Zepeda et al., 2014), cardiomyocyte hypertrophy (Pennanen et al., 2014), and insulin resistance (Del Campo et al., 2014). We also showed that insulin regulates cardiomyocyte metabolism through the control of mitochondrial dynamics (Parra et al., 2014). We also described that the association between two organelles such as ER and mitochondria regulate mitochondrial energetic function, especially as a novel adaptative mechanism during ER stress (Bravo et al., 2011). Interestingly, when the association of both organelles is inhibited by a reduced expression of the protein Mfn2, the metabolic changes are abrogated (Bravo et al., 2011). On the other hand, it has been shown that ERmitochondria coupling also can induce apoptosis through the connection of FIS-1 and Bap31 (an ER membrane protein). This association interacts as a scaffold complex which facilitates cell death signal transfer (Iwasawa et al., 2011).

\section{MITOCHONDRIA, VSMC PROLIFERATION, AND APOPTOSIS}

Mitochondrial metabolism is being recognized as a critical regulator of cell growth and proliferation (Salabei and Hill, 2013). Cells in active division have enhanced glycolysis and glutamine utilization, which provide energy, NADPH, nucleotides, sugars, and amino acids required for cell proliferation (Moncada et al., 2012). In VSMCs, several growth factors, including PDGF-BB, increase mitochondrial activity (Moncada et al., 2012). In pulmonary artery hypertension (PAH) an increased proliferation rate of pulmonary artery smooth muscle cells (PASMCs) is observed. These cells have increased rates of glycolysis (Marsboom et al., 2012) and showed mitochondrial fission (Bonnet et al., 2006; Marsboom et al., 2012), suggesting a direct association between mitochondrial morphology and VSMC phenotypic change (Mitra, 2013). Salabei and Hill, proposed that the PDGF-BB-induced dedifferentiation of VSMCs occurs via two separate processes, i.e., proliferative responses driven by coupled transcriptional and bioenergetic processes, and loss of contractile proteins largely 


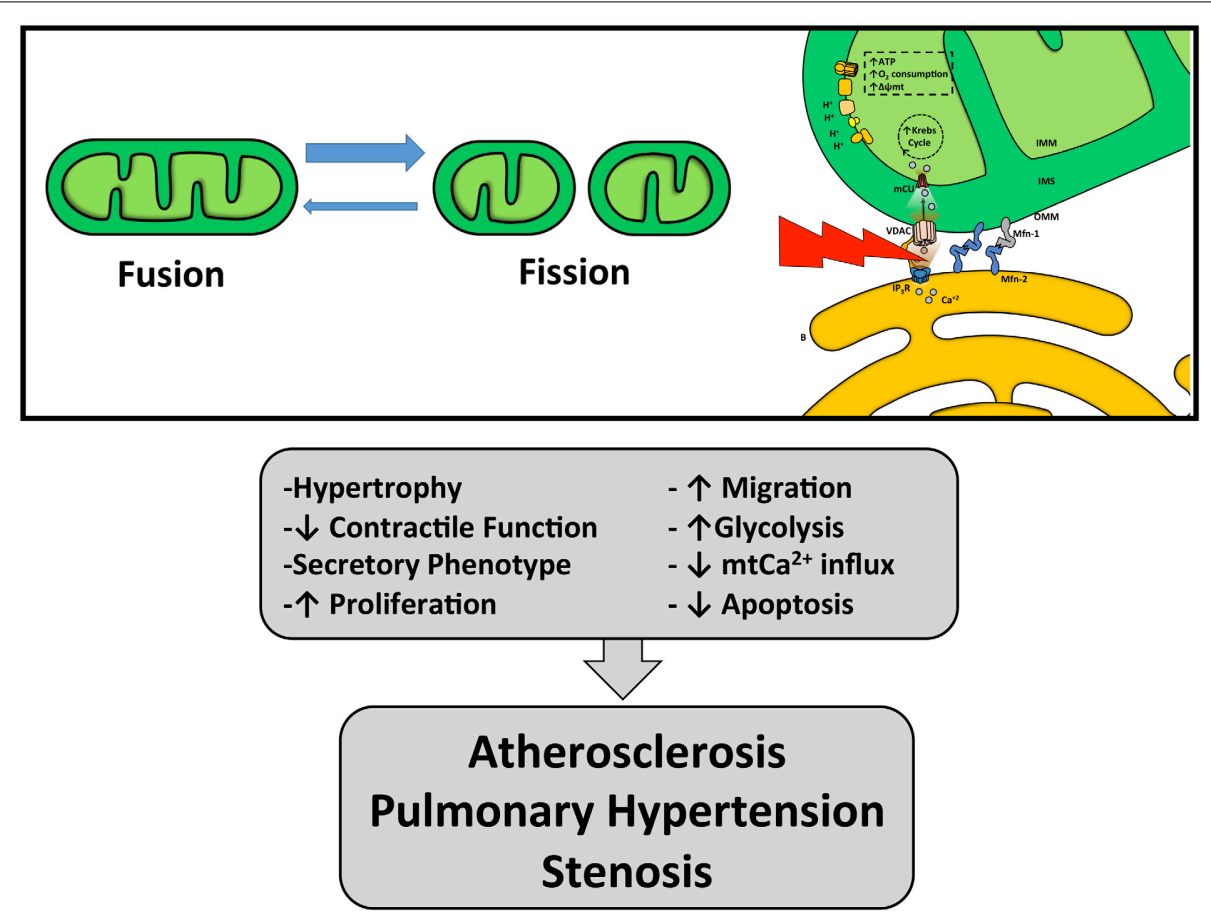

FIGURE 3 | Effects of mitochondria and ER-mitochondrial coupling dysfunction. Unbalance in mitochondrial dynamics and ER-mitochondria uncoupling can lead to VSMC phenotypic switching promoting vascular pathologies such as atherosclerosis, stenosis and pulmonary hypertension.

mediated by autophagy and downregulation of myofilament genes (Salabei and Hill, 2013). Moreover, inhibition of DRP-1 using Mdivi-1, triggers mitochondrial fusion and prevented PDGF-BB-induced upregulation of the cell cycle proteins proliferating cell nuclear antigen (PCNA) and cyclin D1 (Salabei and Hill, 2013).

VSMC proliferation and apoptosis can be also subject to mitochondrial control through the action Mfn-2. In spontaneously hypertensive or atherosclerosis-prone rat VSMC, Mfn-2 levels were diminished (Chen et al., 2004), while overexpression of Mfn2 reduced VSMC proliferation and sensitized to $\mathrm{H}_{2} \mathrm{O}_{2}$ induced apoptosis (Guo et al., 2007a,b). We have shown that the incretin glucagon-like peptide 1 (GLP-1) enhances the functional coupling between ER and mitochondria in VSMC and promotes a faster and higher increase of mitochondrial $\mathrm{Ca}^{2+}$ entry. This enhanced coupling was associated with an increase in mitochondrial metabolism because inhibition of $\mathrm{Ca}^{2+}$ release from ER, using the InsP3R blocker xestospongin $\mathrm{B}$, or the blockade of $\mathrm{Ca}^{2+}$ entry to the mitochondria, using RuRed, abolished GLP1-dependent increase of $\mathrm{O}_{2}$ consumption and $\Delta \psi \mathrm{m}$ (Morales et al., 2014). Therefore, it seems that communication between mitochondria and the ER is an efficient mechanism for promoting mitochondrial metabolism and regulating VSMC phenotype. This enhanced mitochondria-ER coupling is due to an increase of Mfn-2 through a protein kinase A (PKA)-dependent mechanism (Morales et al., 2014). Guo et al. observed that forskolin increases Mfn-2 expression in VSMC (Guo et al., 2007a). Similarly, treatment of mouse Leydig tumor cells with 8Br-cAMP also increases Mfn-2 mRNA and protein levels (Duarte et al., 2012).
Moreover, Mfn-2 has potent metabolic effects. In type 2 diabetes patients, Mfn-2 mRNA and protein levels are diminished, and these levels correlate with increased body mass index and diminished insulin sensitivity (Bach et al., 2005; Hernandez-Alvarez et al., 2010). Furthermore, Mfn-2 depletion causes altered glucose metabolism in vivo, as Mfn-2-KO mice developed glucose intolerance, fasting hyperinsulinemia and altered insulin response (Figure 3) (Sebastian et al., 2012).

Chen et al. showed that Mfn-2 diminishes in highly proliferative VSMC from atherosclerosis-prone or balloon-injured rats and that Mfn-2 overexpression blocks proliferation of neointimal VSMC after balloon injury (Chen et al., 2004). Moreover, Mfn-2 overexpression also suppresses the proliferating effects of oxidized-LDL in rabbit VSMC cultures. The induction of Mfn2 in vivo reduces PCNA positive cells at the neointimal and medial layers from rabbit carotid arteries subjected to air-drying damage (Guo et al., 2007b). These data correlate with the fact that overexpression of Mfn-2 promotes mitochondrial-mediated apoptosis in VSMC cultures, and that Mfn-2 is up-regulated and necessary for $\mathrm{H}_{2} \mathrm{O}_{2}$-induced apoptosis albeit in a mitochondrial fusion-independent mechanism (Guo et al., 2007a). This antiproliferative activity of Mfn- 2 can be negatively regulated by PKA as shown by the decreased PCNA positive cells and neointimal hyperplasia after balloon injury on rats with overexpression of a Mfn-2 S422A mutant form (a variant that cannot be phosphorylated on Ser422, the residue within the PKA-phosphorylation consensus site) (Zhou et al., 2010). The authors suggest that the anti-proliferative/pro-apoptotic activity of Mfn-2 might be a result of down-regulation of the Raf/MAPK pathway or control 
over apoptotic-related proteins, given that cells overexpressing Mfn-2 exhibit lower levels of ERK-1/2 and Akt in response to certain hormones (Chen et al., 2004; Guo et al., 2007a,b) and an increased Bcl-2/Bax ratio (Guo et al., 2007a).

During hypoxia most blood vessels relax but the pulmonary arteries constrict, ultimately becoming occluded by excessive PASMC proliferation, a condition that causes PAH. This is in part because during hypoxia, mitochondria from PASMCs modifies ROS production, which diffuse to the plasma membrane and regulate membrane voltage-dependent potassium channels $(\mathrm{Kv})$ and activate the hypoxia induced factor (HIF-1 $\alpha$ ) signaling pathway, which is redox-sensitive (Michelakis et al., 2002). The inhibition of $\mathrm{Kv}$ channels by mitochondrial ROS cause depolarization, opening of voltage-gated $\mathrm{Ca}^{2+}$ channels, influx of $\mathrm{Ca}^{2+}$, and vasoconstrictive response (Dromparis et al., 2010). However, systemic VSMCs show totally opposite behavior of intracellular $\mathrm{Ca}^{2+}$ in response to hypoxia. Furthermore, there are many differences between PASMC and systemic VSMC mitochondria, such as decreased expression and function of the electron transporter chain complexes I-III and increased expression of superoxide dismutase (SOD). These differences are explained by differences in baseline of mitochondrial ROS between PASMCs and VSMCs, producing the opposite effect during hypoxia, contraction vs. dilatation, respectively (Michelakis et al., 2002).

During the incipient $\mathrm{PAH}$, the mitochondria from PASMC become hyperpolarized and generate less ROS, indices of a metabolic shift. This event is produced during normoxia and can inhibit the Kv channel, which lead to an increase of intracellular $\mathrm{Ca}^{2+}$ levels and generate vessel contraction (Archer et al., 2008). In wild-type mice, these changes are accompanied by an excessive PASMC proliferation, decrease in glucose oxidation and increased glycolysis (Sutendra et al., 2010). When fatty acid oxidation is abolished by deletion of the malonyl-coenzyme A decarboxylase gene, thereby shifting the metabolic balance back to glucose oxidation, the mice do not develop PAH and less or no PASMC proliferation is observed (Sutendra et al., 2010). As mentioned above, the shift away from glucose oxidation toward glycolysis and fatty acid usage in the PASMCs during hypertension is accompanied by alterations in mitochondrial function (Sutendra et al., 2010). Intracellular $\mathrm{Ca}^{2+}$ level increase and glucose oxidation suppression activates transcriptional factors such as nuclear factor of activated T-cells (NFAT) which promotes PASMC proliferation in PAH (Bonnet et al., 2007; Sutendra et al., 2010). In PASMCs, HIF- $1 \alpha$ activation induces cell proliferation and also triggers DRP-1-mediated mitochondrial fission. In vivo, cobalt activates HIF- $1 \alpha$ and induces PAH in rats. Co-administration of Mdivi-1, restores mitochondrial fusion and reduces $\mathrm{PAH}$ (Marsboom et al., 2012). Increased fission observed in PASMCs from PAH results from DRP1 activation by increased cyclin B1/CDK1 activity and accompanies cell cycle progression from G2 to mitosis. DRP-1 inhibition by Mdivi-1 slows proliferation by locking mitochondria in fusion and inhibiting cell-cycle progression, causing G2/M arrest (Marsboom et al., 2012).

When ER stress is induced by chronic normobaric hypoxia in mice PASMCs, mitochondrial $\mathrm{Ca}^{2+}, 2$-oxoglutarate content and pyruvate dehydrogenase activity were decreased, depicting mitochondrial malfunction under these conditions (Sutendra et al.,
2011). Sutendra et al. show that the Nogo-B protein, member of the reticulon family, which is critical in the regulation of tubular ER structure, is induced by hypoxia only in lung vessels, where it disrupts the contacts between the ER and the mitochondria (Sutendra et al., 2011). This alteration disrupts essential mitochondrial functions, causing overgrowth of PASMCs and ultimately PAH. Moreover, Nogo controls the ER shape and inhibits apoptosis during vascular remodeling (Sutendra et al., 2011).

\section{MITOCHONDRIAL DYSFUNCTION IN ATHEROSCLEROSIS}

Damage to the vascular environment by oxidative stress plays a major role in the pathogenesis of atherosclerosis. Under normal conditions, ROS production is controlled by mitochondria and ROS levels are sensed by enzymatic systems and cellular mechanisms. Alteration in ROS production is frequently observed in atherosclerosis. Oxidative stress results in lipid peroxidation and damage of mitochondrial components, including mitochondrial DNA (mtDNA), leading to mitochondrial dysfunction. mtDNA is also affected by mitochondrial fission process. Cleavage of OPA-1 leads to mtDNA loss, respiratory chain deficiency and mitochondrial ROS increase (Duvezin-Caubet et al., 2006; Finsterer, 2007; Parone et al., 2008). ROS increase activates poly(ADPribose) polymerase 1 (PARP-1), enzyme involved in chromatin structure modulation and DNA repair, which increases VSMC and endothelial cell death, facilitating atherosclerosis progression (Virag, 2005). Alteration in mitochondrial anti-oxidant enzyme levels, such as manganese superoxide dismutase (MnSOD), increases mtDNA damage in ApoE ${ }^{-/-}$mice (Ballinger et al., 2002). Furthermore, the extent of mitochondrial, but not nuclear, DNA damage correlates with the development of atherosclerotic VSMC lesions in mice and human aortic tissues (Ballinger et al., 2002). Mitochondrial stress, induced by the mtDNA replication inhibitor, dideoxycytidine, promotes VSMC migration, but not proliferation, suggesting that malfunction of mitochondria may be involved in the plaque stabilization in late-stage atherosclerosis (Ahn et al., 2010). Plaque stabilization is accompanied by increase in VSMC and macrophage apoptosis which promotes induction of pro-coagulation and plaque rupture (Clarke et al., 2006). Also, VSMC functionality depends on mitochondrial genes transcription. VSMC-specific ablation of the mitochondrial transcription factor Tfam results in a diminished contractile response of mesenteric artery rings to phenylephrine (Jawien et al., 2008). Prevention of the upregulation of this transcription factor, observed in carotid artery from rats subjected to balloon injury, might prevent intimal thickening in this type of damaged-artery model (Yoshida et al., 2005). On the other hand, mitochondrial dysfunction in macrophages (changes in ATP production, $\Delta \psi \mathrm{mt}$, and mtDNA damage) alters cholesterol trafficking between mitochondrial membranes facilitating cholesterol accumulation at the interface mitochondrial membrane-ER. This accumulation facilitates interaction between cholesterol and CYP27A1 due to the permeability transition pore (PTP) aperture, generating 27-oxygenated derivatives of cholesterol and accumulation within vascular cells (Allen et al., 2013). These results, among others, suggest that mitochondrial dysfunction is a possible hallmark of vascular diseases. Intervention and modulation of 
these mechanisms will be useful in the treatment and prevention of atherosclerosis progression.

\section{CONCLUDING REMARKS}

VSMC proliferation plays a key role in atherogenesis and restenosis. Mitochondrial dysfunction is a metabolic feature that control VSMC phenotype. Targeting of mitochondrial function with dichloroacetate (Mcmurtry et al., 2004) or trimetazidine (Sutendra et al., 2010) has been successfully used to avoid VSMC or PASMC proliferation in animal models. However, only rapamycin has been tested in human, specifically in drug eluting stents to avoid restenosis (Kang et al., 2011; Testa et al., 2011). Major translational challenges remain in this exciting area, but patients with vascular disease are likely to benefit from these efforts.

\section{ACKNOWLEDGMENTS}

This research was funded in part by Comisión Nacional de Ciencia y Tecnología (CONICYT), Chile (FONDECYT 1110180, FONDECYT 1140329; Anillo ACT1111; FONDAP 15130011). David Mondaca-Ruff and Pablo E. Morales hold a CONICYT PhD fellowship.

\section{REFERENCES}

Ahn, S. Y., Choi, Y. S., Koo, H. J., Jeong, J. H., Park, W. H., Kim, M., et al. (2010). Mitochondrial dysfunction enhances the migration of vascular smooth muscles cells via suppression of Akt phosphorylation. Biochim. Biophys. Acta 1800, 275-281. doi: 10.1016/j.bbagen.2009.09.005

Allen, A. M., Taylor, J. M., and Graham, A. (2013). Mitochondrial (dys)function and regulation of macrophage cholesterol efflux. Clin. Sci. Lond. 124, 509-515. doi: $10.1042 / C S 20120358$

Archer, S. L., Gomberg-Maitland, M., Maitland, M. L., Rich, S., Garcia, J. G., and Weir, E. K. (2008). Mitochondrial metabolism, redox signaling, and fusion: a mitochondria-ROS-HIF-1alpha-Kv1.5 O2-sensing pathway at the intersection of pulmonary hypertension and cancer. Am. J. Physiol. Heart. Circ. Physiol. 294, H570-H578. doi: 10.1152/ajpheart.01324.2007

Bach, D., Naon, D., Pich, S., Soriano, F. X., Vega, N., Rieusset, J., et al. (2005). Expression of Mfn2, the Charcot-Marie-Tooth neuropathy type 2A gene, in human skeletal muscle: effects of type 2 diabetes, obesity, weight loss, and the regulatory role of tumor necrosis factor alpha and interleukin-6. Diabetes 54, 2685-2693. doi: 10.2337/diabetes.54.9.2685

Ballinger, S. W., Patterson, C., Knight-Lozano, C. A., Burow, D. L., Conklin, C. A., Hu, Z., et al. (2002). Mitochondrial integrity and function in atherogenesis. Circulation 106, 544-549. doi: 10.1161/01.CIR.0000023921.93743.89

Baughman, J. M., Perocchi, F., Girgis, H. S., Plovanich, M., Belcher-Timme, C. A., Sancak, Y., et al. (2011). Integrative genomics identifies MCU as an essential component of the mitochondrial calcium uniporter. Nature 476, 341-345. doi: 10.1038/nature 10234

Berridge, M. J., Bootman, M. D., and Roderick, H. L. (2003). Calcium signalling: dynamics, homeostasis and remodelling. Nat. Rev. Mol. Cell Biol. 4, 517-529. doi: $10.1038 / \mathrm{nrm} 1155$

Bola, B., and Allan, V. (2009). How and why does the endoplasmic reticulum move? Biochem. Soc. Trans. 37, 961-965. doi: 10.1042/BST0370961

Boldogh, I. R., and Pon, L. A. (2007). Mitochondria on the move. Trends Cell Biol. 17, 502-510. doi: 10.1016/j.tcb.2007.07.008

Bonnet, S., Michelakis, E. D., Porter, C. J., Andrade-Navarro, M. A., Thebaud, B., Bonnet, S., et al. (2006). An abnormal mitochondrial-hypoxia inducible factor-1alpha-Kv channel pathway disrupts oxygen sensing and triggers pulmonary arterial hypertension in fawn hooded rats: similarities to human pulmonary arterial hypertension. Circulation 113, 2630-2641. doi: 10.1161/CIRCULATIONAHA.105.609008

Bonnet, S., Rochefort, G., Sutendra, G., Archer, S. L., Haromy, A., Webster, L., et al. (2007). The nuclear factor of activated $\mathrm{T}$ cells in pulmonary arterial hypertension can be therapeutically targeted. Proc. Natl. Acad. Sci. U.S.A. 104, 11418-11423. doi: 10.1073/pnas.0610467104
Bossy-Wetzel, E., Barsoum, M. J., Godzik, A., Schwarzenbacher, R., and Lipton, S. A. (2003). Mitochondrial fission in apoptosis, neurodegeneration and aging. Curr. Opin. Cell Biol. 15, 706-716. doi: 10.1016/j.ceb.2003.10.015

Bravo, R., Vicencio, J. M., Parra, V., Troncoso, R., Munoz, J. P., Bui, M., et al. (2011), Increased ER-mitochondrial coupling promotes mitochondrial respiration and bioenergetics during early phases of ER stress. J. Cell Sci. 124, 2143-2152. doi: $10.1242 /$ jcs. 080762

Bravo-Sagua, R., Rodriguez, A. E., Kuzmicic, J., Gutierrez, T., Lopez-Crisosto, C., Quiroga, C., et al. (2013). Cell death and survival through the endoplasmic reticulum-mitochondrial axis. Curr. Mol. Med. 13, 317-329. doi: $10.2174 / 156652413804810781$

Bravo-Sagua, R., Torrealba, N., Paredes, F., Morales, P. E., Pennanen, C., LopezCrisosto, C., et al. (2014). Organelle communication: signaling crossroads between homeostasis and disease. Int. J. Biochem. Cell Biol. 50, 55-59. doi: 10.1016/j.biocel.2014.01.019

Butler, T. M., and Siegman, M. J. (1985). High-energy phosphate metabolism in vascular smooth muscle. Annu. Rev. Physiol. 47, 629-643. doi: 10.1146/annurev.ph.47.030185.003213

Campbell, G. R., and Campbell, J. H. (1985). Smooth muscle phenotypic changes in arterial wall homeostasis: implications for the pathogenesis of atherosclerosis. Exp. Mol. Pathol. 42, 139-162. doi: 10.1016/0014-4800(85)90023-1

Cardenas, C., Miller, R. A., Smith, I., Bui, T., Molgo, J., Muller, M., et al. (2010). Essential regulation of cell bioenergetics by constitutive InsP3 receptor Ca2+ transfer to mitochondria. Cell 142, 270-283. doi: 10.1016/j.cell.2010.06.007

Carlucci, A., Lignitto, L., and Feliciello, A. (2008). Control of mitochondria dynamics and oxidative metabolism by cAMP, AKAPs and the proteasome. Trends Cell Biol. 18, 604-613. doi: 10.1016/j.tcb.2008.09.006

Cecchettini, A., Rocchiccioli, S., Boccardi, C., and Citti, L. (2011). Vascular smoothmuscle-cell activation: proteomics point of view. Int. Rev. Cell Mol. Biol. 288, 43-99. doi: 10.1016/B978-0-12-386041-5.00002-9

Cerveny, K. L., Tamura, Y., Zhang, Z., Jensen, R. E., and Sesaki, H. (2007). Regulation of mitochondrial fusion and division. Trends Cell Biol. 17, 563-569. doi: 10.1016/j.tcb.2007.08.006

Chen, H., and Chan, D. C. (2005). Emerging functions of mammalian mitochondrial fusion and fission. Hum. Mol. Genet. 14, R283-R289. doi: 10.1093/hmg/ddi270

Chen, H., Detmer, S. A., Ewald, A. J., Griffin, E. E., Fraser, S. E., and Chan, D. C. (2003). Mitofusins Mfn1 and Mfn2 coordinately regulate mitochondrial fusion and are essential for embryonic development. J. Cell Biol. 160, 189-200. doi: $10.1083 / j \mathrm{cb} .200211046$

Chen, K. H., Guo, X., Ma, D., Guo, Y., Li, Q., Yang, D., et al. (2004). Dysregulation of HSG triggers vascular proliferative disorders. Nat. Cell Biol. 6, 872-883. doi: $10.1038 /$ ncb1161

Chen, L., Gong, Q., Stice, J. P., and Knowlton, A. A. (2009). Mitochondrial OPA1, apoptosis, and heart failure. Cardiovasc. Res. 84, 91-99. doi: 10.1093/cvr/cvp181

Clarke, M. C., Figg, N., Maguire, J. J., Davenport, A. P., Goddard, M., Littlewood, T. D., et al. (2006). Apoptosis of vascular smooth muscle cells induces features of plaque vulnerability in atherosclerosis. Nat. Med. 12, 1075-1080. doi: $10.1038 / \mathrm{nm} 1459$

Csordas, G., Madesh, M., Antonsson, B., and Hajnoczky, G. (2002). tcBid promotes $\mathrm{Ca}(2+)$ signal propagation to the mitochondria: control of $\mathrm{Ca}(2+)$ permeation through the outer mitochondrial membrane. EMBO J. 21, 2198-2206. doi: 10.1093/emboj/21.9.2198

Csordas, G., Thomas, A. P., and Hajnoczky, G. (1999). Quasi-synaptic calcium signal transmission between endoplasmic reticulum and mitochondria. $E M B O J$. 18, 96-108. doi: 10.1093/emboj/18.1.96

De Brito, O. M., and Scorrano, L. (2008). Mitofusin 2 tethers endoplasmic reticulum to mitochondria. Nature 456, 605-610. doi: 10.1038/nature07534

Del Campo, A., Parra, V., Vasquez-Trincado, C., Gutierrez, T., Morales, P. E., Lopez-Crisosto, C., et al. (2014). Mitochondrial fragmentation impairs insulindependent glucose uptake by modulating Akt activity through mitochondrial Ca2+ uptake. Am. J. Physiol. Endocrinol. Metab. 306, E1-E13. doi: 10.1152/ajpendo.00146.2013

Denton, R. M. (2009). Regulation of mitochondrial dehydrogenases by calcium ions. Biochim. Biophys. Acta 1787, 1309-1316. doi: 10.1016/j.bbabio.2009.01.005

Denton, R. M., and Mccormack, J. G. (1990). Ca2+ as a second messenger within mitochondria of the heart and other tissues. Annu. Rev. Physiol. 52, 451-466. doi: 10.1146/annurev.ph.52.030190.002315 
De Stefani, D., Raffaello, A., Teardo, E., Szabo, I., and Rizzuto, R. (2011). A forty-kilodalton protein of the inner membrane is the mitochondrial calcium uniporter. Nature 476, 336-340. doi: 10.1038/nature10230

Dromparis, P., Sutendra, G., and Michelakis, E. D. (2010). The role of mitochondria in pulmonary vascular remodeling. J. Mol. Med. Berl. 88, 1003-1010. doi: 10.1007/s00109-010-0670-x

Duarte, A., Poderoso, C., Cooke, M., Soria, G., Cornejo Maciel, F., Gottifredi, V., et al. (2012). Mitochondrial fusion is essential for steroid biosynthesis. PLoS ONE 7:e45829. doi: 10.1371/journal.pone.0045829

Duvezin-Caubet, S., Jagasia, R., Wagener, J., Hofmann, S., Trifunovic, A., Hansson, A., et al. (2006). Proteolytic processing of OPAl links mitochondrial dysfunction to alterations in mitochondrial morphology. J. Biol. Chem. 281, 37972-37979. doi: 10.1074/jbc.M606059200

Dykens, J. A. (1994). Isolated cerebral and cerebellar mitochondria produce free radicals when exposed to elevated $\mathrm{CA} 2+$ and $\mathrm{Na}+$ : implications for neurodegeneration. J. Neurochem. 63, 584-591. doi: 10.1046/j.1471-4159.1994.63020584.x

English, A. R., and Voeltz, G. K. (2013). Endoplasmic reticulum structure and interconnections with other organelles. Cold Spring Harb. Perspect. Biol. 5:a013227. doi: 10.1101/cshperspect.a013227

Finsterer, J. (2007). Is atherosclerosis a mitochondrial disorder? Vasa 36, 229-240. doi: 10.1024/0301-1526.36.4.229

Griffiths, E. J., and Rutter, G. A. (2009). Mitochondrial calcium as a key regulator of mitochondrial ATP production in mammalian cells. Biochim. Biophys. Acta 1787, 1324-1333. doi: 10.1016/j.bbabio.2009.01.019

Guo, X., Chen, K. H., Guo, Y., Liao, H., Tang, J., and Xiao, R. P. (2007a). Mitofusin 2 triggers vascular smooth muscle cell apoptosis via mitochondrial death pathway. Circ. Res. 101, 1113-1122. doi: 10.1161/CIRCRESAHA.107.157644

Guo, Y. H., Chen, K., Gao, W., Li, Q., Chen, L., Wang, G. S., et al. (2007b). Overexpression of Mitofusin 2 inhibited oxidized low-density lipoprotein induced vascular smooth muscle cell proliferation and reduced atherosclerotic lesion formation in rabbit. Biochem. Biophys. Res. Commun. 363, 411-417. doi: 10.1016/j.bbrc.2007.08.191

Hernandez-Alvarez, M. I., Thabit, H., Burns, N., Shah, S., Brema, I., Hatunic, M., et al. (2010). Subjects with early-onset type 2 diabetes show defective activation of the skeletal muscle PGC-1\{alpha\}/Mitofusin-2 regulatory pathway in response to physical activity. Diabetes Care 33, 645-651. doi: 10.2337/dc09-1305

Hoppins, S. (2014). The regulation of mitochondrial dynamics. Curr. Opin. Cell Biol. 29, 46-52. doi: 10.1016/j.ceb.2014.03.005

Ishihara, N., Nomura, M., Jofuku, A., Kato, H., Suzuki, S. O., Masuda, K., et al. (2009). Mitochondrial fission factor Drpl is essential for embryonic development and synapse formation in mice. Nat. Cell Biol. 11, 958-966. doi: $10.1038 /$ ncb 1907

Iwasawa, R., Mahul-Mellier, A. L., Datler, C., Pazarentzos, E., and Grimm, S. (2011). Fis1 and Bap31 bridge the mitochondria-ER interface to establish a platform for apoptosis induction. EMBO J. 30, 556-568. doi: 10.1038/emboj. 2010.346

James, D. I., Parone, P. A., Mattenberger, Y., and Martinou, J. C. (2003). hFis1, a novel component of the mammalian mitochondrial fission machinery. J. Biol. Chem. 278, 36373-36379. doi: 10.1074/jbc.M303758200

Jawien, J., Bian, Z., Sheikine, Y., Olofsson, P. S., Pang, Y., Edholm, T., et al. (2008). Abrogation of mitochondrial transcription in smooth muscle cells impairs smooth muscle contractility and vascular tone. J. Physiol. Pharmacol. 59, 239-252.

Joyal, J. L., Hagen, T., and Aprille, J. R. (1995). Intramitochondrial protein synthesis is regulated by matrix adenine nucleotide content and requires calcium. Arch. Biochem. Biophys. 319, 322-330. doi: 10.1006/abbi.1995.1300

Kang, W. C., Park, Y. M., Shin, K. C., Moon, C. I., Lee, K., Han, S. H., et al. (2011). Comparison of edge vascular response after sirolimus- and paclitaxel-eluting stent implantation. Int. J. Cardiol. 165, 46-50. doi: 10.1016/j.ijcard.2011.07.108

Kirichok, Y., Krapivinsky, G., and Clapham, D. E. (2004). The mitochondrial calcium uniporter is a highly selective ion channel. Nature 427, 360-364. doi: 10.1038 /nature02246

Korobova, F., Ramabhadran, V., and Higgs, H. N. (2013). An actin-dependent step in mitochondrial fission mediated by the ER-associated formin INF2. Science 339, 464-467. doi: 10.1126/science. 1228360

Kuzmicic, J., Del Campo, A., Lopez-Crisosto, C., Morales, P. E., Pennanen, C., Bravo-Sagua, R., et al. (2011). Mitochondrial dynamics: a potential new therapeutic target for heart failure. Rev. Esp. Cardiol. Engl. Ed. 64, 916-923. doi: 10.1016/j.rec.2011.05.022
Kuznetsov, A. V., Hermann, M., Saks, V., Hengster, P., and Margreiter, R. (2009). The cell-type specificity of mitochondrial dynamics. Int. J. Biochem. Cell Biol. 41, 1928-1939. doi: 10.1016/j.biocel.2009.03.007

Lee, A. C., Xu, X., and Colombini, M. (1996). The role of pyridine dinucleotides in regulating the permeability of the mitochondrial outer membrane. J. Biol. Chem. 271, 26724-26731. doi: 10.1074/jbc.271.43.26724

Liu, T., and O'Rourke, B. (2009). Regulation of mitochondrial Ca2+ and its effects on energetics and redox balance in normal and failing heart. J. Bioenerg. Biomembr. 41, 127-132. doi: 10.1007/s10863-009-9216-8

Malka, F., Guillery, O., Cifuentes-Diaz, C., Guillou, E., Belenguer, P., Lombes, A., et al. (2005). Separate fusion of outer and inner mitochondrial membranes. EMBO Rep. 6, 853-859. doi: 10.1038/sj.embor.7400488

Marsboom, G., Toth, P. T., Ryan, J. J., Hong, Z., Wu, X., Fang, Y. H., et al. (2012). Dynamin-related protein 1-mediated mitochondrial mitotic fission permits hyperproliferation of vascular smooth muscle cells and offers a novel therapeutic target in pulmonary hypertension. Circ. Res. 110, 1484-1497. doi: 10.1161/CIRCRESAHA.111.263848

Mcmurtry, M. S., Bonnet, S., Wu, X., Dyck, J. R., Haromy, A., Hashimoto, K., et al. (2004). Dichloroacetate prevents and reverses pulmonary hypertension by inducing pulmonary artery smooth muscle cell apoptosis. Circ. Res. 95, 830-840. doi: 10.1161/01.RES.0000145360.16770.9f

Michelakis, E. D., Hampl, V., Nsair, A., Wu, X., Harry, G., Haromy, A., et al. (2002). Diversity in mitochondrial function explains differences in vascular oxygen sensing. Circ. Res. 90, 1307-1315. doi: 10.1161/01.RES.0000024689.07590.C2

Mishra, P., and Chan, D. C. (2014). Mitochondrial dynamics and inheritance during cell division, development and disease. Nat. Rev. Mol. Cell Biol. 15, 634-646. doi: $10.1038 / \mathrm{nrm} 3877$

Mitra, K. (2013). Mitochondrial fission-fusion as an emerging key regulator of cell proliferation and differentiation. Bioessays 35, 955-964. doi: 10.1002/bies.201300011

Moncada, S., Higgs, E. A., and Colombo, S. L. (2012). Fulfilling the metabolic requirements for cell proliferation. Biochem. J. 446, 1-7. doi: 10.1042/BJ20120427

Morales, P. E., Torres, G., Sotomayor-Flores, C., Pena-Oyarzun, D., Rivera-Mejias, P., Paredes, F., et al. (2014). GLP-1 promotes mitochondrial metabolism in vascular smooth muscle cells by enhancing endoplasmic reticulummitochondria coupling. Biochem. Biophys. Res. Commun. 446, 410-416. doi: 10.1016/j.bbrc.2014.03.004

Naon, D., and Scorrano, L. (2014). At the right distance: ER-mitochondria juxtaposition in cell life and death. Biochim. Biophys. Acta 1843, 2184-2194. doi: 10.1016/j.bbamcr.2014.05.011

Nasrallah, C. M., and Horvath, T. L. (2014). Mitochondrial dynamics in the central regulation of metabolism. Nat. Rev. Endocrinol. 10, 650-658. doi: 10.1038/nrendo.2014.160

Palmer, C. S., Elgass, K. D., Parton, R. G., Osellame, L. D., Stojanovski, D., and Ryan, M. T. (2013). Adaptor proteins MiD49 and MiD51 can act independently of Mff and Fis1 in Drpl recruitment and are specific for mitochondrial fission. J. Biol. Chem. 288, 27584-27593. doi: 10.1074/jbc.M113.479873

Parone, P. A., Da Cruz, S., Tondera, D., Mattenberger, Y., James, D. I., Maechler, P., et al. (2008). Preventing mitochondrial fission impairs mitochondrial function and leads to loss of mitochondrial DNA. PLOS ONE 3:e3257. doi: 10.1371/journal.pone.0003257

Parra, V., Eisner, V., Chiong, M., Criollo, A., Moraga, F., Garcia, A., et al. (2008). Changes in mitochondrial dynamics during ceramide-induced cardiomyocyte early apoptosis. Cardiovasc. Res. 77, 387-397. doi: 10.1093/cvr/cvm029

Parra, V., Verdejo, H., Del Campo, A., Pennanen, C., Kuzmicic, J., Iglewski, M., et al. (2011). The complex interplay between mitochondrial dynamics and cardiac metabolism. J. Bioenerg. Biomembr. 43, 47-51. doi: 10.1007/s10863-011-9332-0

Parra, V., Verdejo, H. E., Iglewski, M., Del Campo, A., Troncoso, R., Jones, D., et al. (2014). Insulin stimulates mitochondrial fusion and function in cardiomyocytes via the Akt-mTOR-NFkappaB-Opa-1 signaling pathway. Diabetes 63, 75-88. doi: $10.2337 / \mathrm{db} 13-0340$

Paul, R. J. (1983). Functional compartmentalization of oxidative and glycolytic metabolism in vascular smooth muscle. Am. J. Physiol. Cell Physiol. 244, C399-C409.

Pennanen, C., Parra, V., Lopez-Crisosto, C., Morales, P. E., Del Campo, A., Gutierrez, T., et al. (2014). Mitochondrial fission is required for cardiomyocyte hypertrophy via a Ca2+-calcineurin signalling pathway. J. Cell Sci. 127, 2659-2671. doi: 10.1242/jcs.139394 
Pich, S., Bach, D., Briones, P., Liesa, M., Camps, M., Testar, X., et al. (2005). The Charcot-Marie-Tooth type 2A gene product, Mfn2, up-regulates fuel oxidation through expression of OXPHOS system. Hum. Mol. Genet. 14, 1405-1415. doi: 10.1093/hmg/ddil49

Rapizzi, E., Pinton, P., Szabadkai, G., Wieckowski, M. R., Vandecasteele, G., Baird, G., et al. (2002). Recombinant expression of the voltage-dependent anion channel enhances the transfer of $\mathrm{Ca} 2+$ microdomains to mitochondria. J. Cell Biol. 159, 613-624. doi: 10.1083/jcb.200205091

Rizzuto, R., De Stefani, D., Raffaello, A., and Mammucari, C. (2012). Mitochondria as sensors and regulators of calcium signalling. Nat. Rev. Mol. Cell Biol. 13, 566-578. doi: 10.1038/nrm3412

Rowland, A. A., and Voeltz, G. K. (2012). Endoplasmic reticulum-mitochondria contacts: function of the junction. Nat. Rev. Mol. Cell Biol. 13, 607-625. doi: $10.1038 / \mathrm{nrm} 3440$

Rzucidlo, E. M., Martin, K. A., and Powell, R. J. (2007). Regulation of vascular smooth muscle cell differentiation. J. Vasc. Surg. 45(Suppl. A), A25-A32. doi: 10.1016/j.jvs.2007.03.001

Salabei, J. K., and Hill, B. G. (2013). Mitochondrial fission induced by plateletderived growth factor regulates vascular smooth muscle cell bioenergetics and cell proliferation. Redox Biol. 1, 542-551. doi: 10.1016/j.redox.2013.10.011

Scheffler, I. E. (2001). Mitochondria make a come back. Adv. Drug Deliv. Rev. 49, 3-26. doi: 10.1016/S0169-409X(01)00123-5

Schwarzer, C., Barnikol-Watanabe, S., Thinnes, F. P., and Hilschmann, N. (2002). Voltage-dependent anion-selective channel (VDAC) interacts with the dynein light chain Tctexl and the heat-shock protein PBP74. Int. J. Biochem. Cell Biol. 34, 1059-1070. doi: 10.1016/S1357-2725(02)00026-2

Sebastian, D., Hernandez-Alvarez, M. I., Segales, J., Sorianello, E., Munoz, J. P., Sala, D., et al. (2012). Mitofusin 2 (Mfn2) links mitochondrial and endoplasmic reticulum function with insulin signaling and is essential for normal glucose homeostasis. Proc. Natl. Acad. Sci. U.S.A. 109, 5523-5528. doi: 10.1073/pnas.1108220109

Sharma, N., Okere, I. C., Brunengraber, D. Z., Mcelfresh, T. A., King, K. L., Sterk, J. P., et al. (2005). Regulation of pyruvate dehydrogenase activity and citric acid cycle intermediates during high cardiac power generation. J. Physiol. 562, 593-603. doi: 10.1113/jphysiol.2004.075713

Soubannier, V., and Mcbride, H. M. (2009). Positioning mitochondrial plasticity within cellular signaling cascades. Biochim. Biophys. Acta 1793, 154-170. doi: 10.1016/j.bbamcr.2008.07.008

Sutendra, G., Bonnet, S., Rochefort, G., Haromy, A., Folmes, K. D., Lopaschuk, G. D., et al. (2010). Fatty acid oxidation and malonyl-CoA decarboxylase in the vascular remodeling of pulmonary hypertension. Sci. Transl. Med. 2, 44ra58. doi: 10.1126/scitranslmed.3001327

Sutendra, G., Dromparis, P., Wright, P., Bonnet, S., Haromy, A., Hao, Z., et al. (2011). The role of Nogo and the mitochondria-endoplasmic reticulum unit in pulmonary hypertension. Sci. Transl. Med. 3, 88ra55. doi: 10.1126/scitranslmed.3002194

Testa, L., Latini, R. A., Pizzocri, S., Lanotte, S., Agnifili, M., Laudisa, M. L., et al. (2011). Multi-Link Vision stent vs. first-generation drug-eluting stents: systematic review and meta-analysis. QJM 104, 1025-1034. doi: 10.1093/qjmed/ hor 138

Vance, J. E. (2014). MAM (mitochondria-associated membranes) in mammalian cells: lipids and beyond. Biochim. Biophys. Acta 1841, 595-609. doi: 10.1016/j.bbalip.2013.11.014
Vander Heiden, M. G., Cantley, L. C., and Thompson, C. B. (2009). Understanding the Warburg effect: the metabolic requirements of cell proliferation. Science 324, 1029-1033. doi: 10.1126/science.1160809

Vander Heiden, M. G., Li, X. X., Gottleib, E., Hill, R. B., Thompson, C. B., and Colombini, M. (2001). Bcl-xL promotes the open configuration of the voltage-dependent anion channel and metabolite passage through the outer mitochondrial membrane. J. Biol. Chem. 276, 19414-19419. doi: 10.1074/jbc.M101590200

Virag, L. (2005). Structure and function of poly(ADP-ribose) polymerase-1: role in oxidative stress-related pathologies. Curr. Vasc. Pharmacol. 3, 209-214. doi: $10.2174 / 1570161054368625$

Wakabayashi, J., Zhang, Z., Wakabayashi, N., Tamura, Y., Fukaya, M., Kensler, T. W., et al. (2009). The dynamin-related GTPase Drpl is required for embryonic and brain development in mice. J. Cell Biol. 186, 805-816. doi: 10.1083/jcb.200903065

Yoshida, T., Azuma, H., Aihara, K., Fujimura, M., Akaike, M., Mitsui, T., et al. (2005). Vascular smooth muscle cell proliferation is dependent upon upregulation of mitochondrial transcription factor A (mtTFA) expression in injured rat carotid artery. Atherosclerosis 178, 39-47. doi: 10.1016/j.atherosclerosis.2004.08.029

Yu, E. P., and Bennett, M. R. (2014). Mitochondrial DNA damage and atherosclerosis. Trends Endocrinol. Metab. 25, 481-487. doi: 10.1016/j.tem.2014.06.008

Zepeda, R., Kuzmicic, J., Parra, V., Troncoso, R., Pennanen, C., Riquelme, J. A., et al. (2014). Drp1 loss-of-function reduces cardiomyocyte oxygen-dependence protecting the heart from ischemia-reperfusion injury. J. Cardiovasc. Pharmacol. 63, 477-487. doi: 10.1097/FJC.0000000000000071

Zhou, W., Chen, K. H., Cao, W., Zeng, J., Liao, H., Zhao, L., et al. (2010). Mutation of the protein kinase A phosphorylation site influences the anti-proliferative activity of mitofusin 2. Atherosclerosis 211, 216-223. doi: 10.1016/j.atherosclerosis.2010.02.012

Zorzano, A., Liesa, M., Sebastian, D., Segales, J., and Palacin, M. (2010). Mitochondrial fusion proteins: dual regulators of morphology and metabolism. Semin. Cell Dev. Biol. 21, 566-574. doi: 10.1016/j.semcdb.2010.01.002

Conflict of Interest Statement: The authors declare that the research was conducted in the absence of any commercial or financial relationships that could be construed as a potential conflict of interest.

Received: 29 August 2014; accepted: 28 November 2014; published online: 15 December 2014.

Citation: Chiong M, Cartes-Saavedra B, Norambuena-Soto I, Mondaca-Ruff D, Morales PE, García-Miguel M and Mellado R (2014) Mitochondrial metabolism and the control of vascular smooth muscle cell proliferation. Front. Cell Dev. Biol. 2:72. doi: 10.3389/fcell.2014.00072

This article was submitted to Molecular Medicine, a section of the journal Frontiers in Cell and Developmental Biology.

Copyright (c) 2014 Chiong, Cartes-Saavedra, Norambuena-Soto, Mondaca-Ruff, Morales, García-Miguel and Mellado. This is an open-access article distributed under the terms of the Creative Commons Attribution License (CC BY). The use, distribution or reproduction in other forums is permitted, provided the original author(s) or licensor are credited and that the original publication in this journal is cited, in accordance with accepted academic practice. No use, distribution or reproduction is permitted which does not comply with these terms. 